\title{
Minimizing HCN in DIC/Oxyma mediated amide bond forming reactions
}

Marion Erny, ${ }^{\mathrm{a}, \mathrm{c}}$ Marika Lundqvist, ${ }^{\mathrm{b}}$ Jon H. Rasmussen, ${ }^{\mathrm{b}}$ Olivier Ludemann-Hombourger, ${ }^{\mathrm{a}}$ Frédéric Bihel ${ }^{\mathrm{c}}$ and Jan Pawlas ${ }^{\mathrm{b},{ }^{*}}$

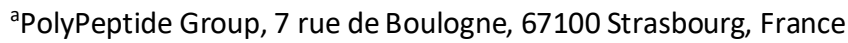

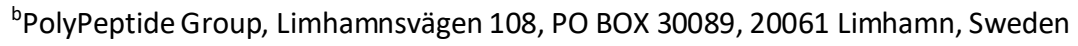

'Laboratoire d'Innovation Thérapeutique, UMR7200, CNRS, Université de Strasbourg, Faculty of Pharmacy, 74 Route du Rhin, 67401 Illkirch-Graffenstaden, France

${ }^{*}$ Corresponding author. E-mail: jan.pawlas@polypeptide.com

\section{Abstract}

Aiming at advancing protocols for safer, environmentally sensible peptide synthesis we report our findings with regards to the occurrence of hydrogen cyanide ( $\mathrm{HCN}$, prussic acid) in amide bond forming reactions mediated by diisopropylcarbodiimide (DIC) and ethyl (hydroxyimino)cyanoacetate (Oxyma). We have determined that HCN is always formed in amide bond forming reactions on solid support in N,N-dimethylformamide (DMF) when employing DIC/Oxyma. In an attempt to minimize the formation of prussic acid by means of preventing the linear DIC/Oxyma adduct $\mathbf{2}$ from cyclizing to oxadiazole $\mathbf{3}$ and in turn releasing $\mathrm{HCN}$, we evaluated a series of greener solvents such as N-butylpyrrolidinone (NBP), NBP/ethyl acetate (EtOAc, 1:1), methyl 5-(dimethylamino)-2-methyl-5-oxopentanoate (PolarClean, PC), and PC/EtOAc (1:1). We found that the ratio between $\mathbf{2}$ and $\mathbf{3}$ greatly depends on the solvent used and consequently, we further examined DMF, NBP, NBP/EtOAC (1:1) and NBP/EtOAC (1:4) as solvents for DIC/Oxyma mediated amidations on solid support and in solution. We found that using carboxylic acid/Oxyma/DIC in a 1:1:1 ratio the rate of HCN formation decreases in the following order DMF $>N B P>N B P / E t O A C(1: 1)>N B P / E t O A C \quad(1: 4)$ while the reaction rate increases in order of $D M F \sim N B P<N B P / E t O A C(1: 1)<N B P / E t O A C(1: 4)$. Of the solvents examined, the NBP/EtOAC (1:4) mixture gave the lowest rate of $\mathrm{HCN}$ formation and the highest rate of amide bond formation both in solution and on solid support. As altering the solvent for DIC/Oxyma mediated amidations resulted in suppressing HCN rather than its full elimination we evaluated the concept of in situ scavenging of the HCN formed. We carried out DIC/Oxyma mediated amidation of Fmoc-Gly-OH + (S)-(-)-1-phenylethylamine in DMF- $d_{7}$ with 0,5 and 10 equiv of dimethyl trisulfide (DMTS) as HCN scavenger. The formation of HCN and rate of amidation was monitored by ${ }^{1} \mathrm{H}$ NMR, revealing that DMTS scavenges $\mathrm{HCN}$ without inhibiting the rate of amidation. DIC/Oxyma mediated amidations of Fmoc-Ser(t-Bu)-OH with (S)-(-)-1-phenylethylamine in DMF and NBP/EtOAc (1:4) with and without 10 equiv of DMTS were carried out and found to be comparable.

TOC Graphic

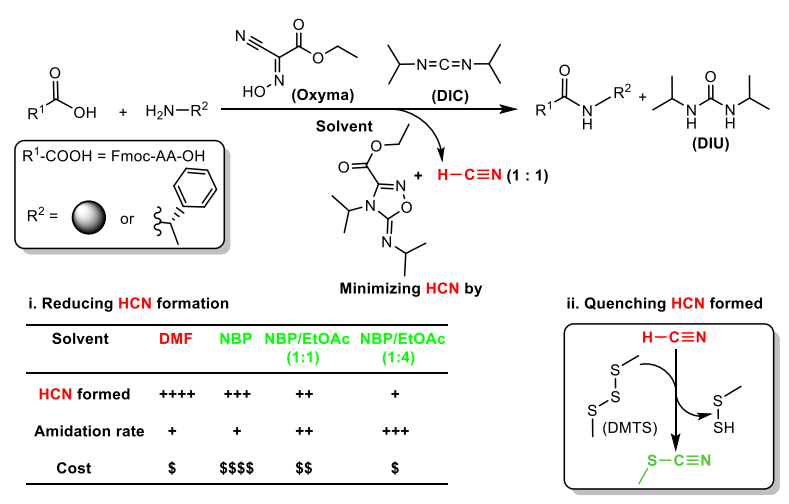


The focus on sustainable chemistry as a means to contribute to the advancement of United Nations' Sustainable Development Goals has been increasing in recent years. ${ }^{1}$ Adhering to the principles of Green Chemistry ${ }^{2}$ is a key prerequisite for the development of sustainable chemical processes and due to the essential role of amide bonds in chemistry and biology, ${ }^{3}$ greening of methods for the construction of amide bonds has received tremendous interest as of late, ${ }^{4}$ for example within the areas of catalytic ${ }^{5}$ and non-classical ${ }^{6}$ amide bond forming reactions. Nevertheless, a vast majority of amide bonds are still being made using a wide range of conventional coupling reagents. ${ }^{7}$ In fact, evaluating various environment, health and safety (EHS) aspects of using these coupling agents has seen growing attention as well. ${ }^{8}$ Among the plethora of reagents available for amide bond formation, the combination of $\mathrm{DIC}^{9}$ and $\mathrm{Oxyma}{ }^{10}$ has become established as one of the most widely used for efficient amide and peptide synthesis. ${ }^{11}$ With regards to EHS considerations, DIC, which in itself promotes an efficient formation of amide bonds, ${ }^{12}$ but is mostly used in the presence of various coupling additives, ${ }^{7,13}$ has been deemed as one of the most preferred coupling reagents from the process safety standpoint. ${ }^{8 a}$ Oxyma has been reported to constitute a highly efficient, greener ${ }^{4 b}$ and safer alternative ${ }^{14}$ to the hydroxybenzotriazole based coupling additives ${ }^{7}$ which are also highly efficient racemization suppressants albeit their use in peptide synthesis has decreased considerably since explosive properties and classification of benzotriazole based coupling agents has been demonstrated. ${ }^{15}$ Recently, McFarland et al. (Eli Lilly) reported that reacting DIC with Oxyma, either alone or in the presence of an amino acid (AA) component, leads to the formation of an oxadiazole byproduct 3 accompanied by an equimolar amount of HCN (Fig. 1a). ${ }^{16}$ Although HCN has conceivably played a key role in the emergence of all life in the early days of our planet, ${ }^{17}$ nowadays $\mathrm{HCN}$ is mostly associated with its very high toxicity. ${ }^{18}$ In fact, the National Institute for Occupational Safety and Health (NIOSH) defines a level of 50 ppm of HCN gas as immediately dangerous to life or health. ${ }^{19}$ The recent report by the scientists at Eli Lilly on occurrence of HCN upon reacting DIC with Oxyma ${ }^{16}$ has therefore warranted a careful evaluation of possible EHS consequences of using DIC/Oxyma in amide and peptide synthesis. On this topic, it was recently noted by Albericio and coworkers ${ }^{20}$ that the occurrence of this side reaction in the presence of an amino component, which corresponds to the real case in peptide synthesis, has not been reported. In fact, these authors indicated that the issue of $\mathrm{HCN}$ formation could be less pronounced than what was reported in the instances in which DIC/Oxyma was reacted in the absence of an amine. ${ }^{16}$

In connection with our ongoing interest and efforts in greening peptide chemistry ${ }^{21}$ together with the fact that large quantities of DIC/Oxyma are used in the manufacturing of peptide therapeutics, these reports prompted us to investigate the extent of HCN formation under the conditions of DIC/Oxyma mediated amide bond forming reactions, and to identify possible courses of actions for mitigation. (Fig. 1b). 


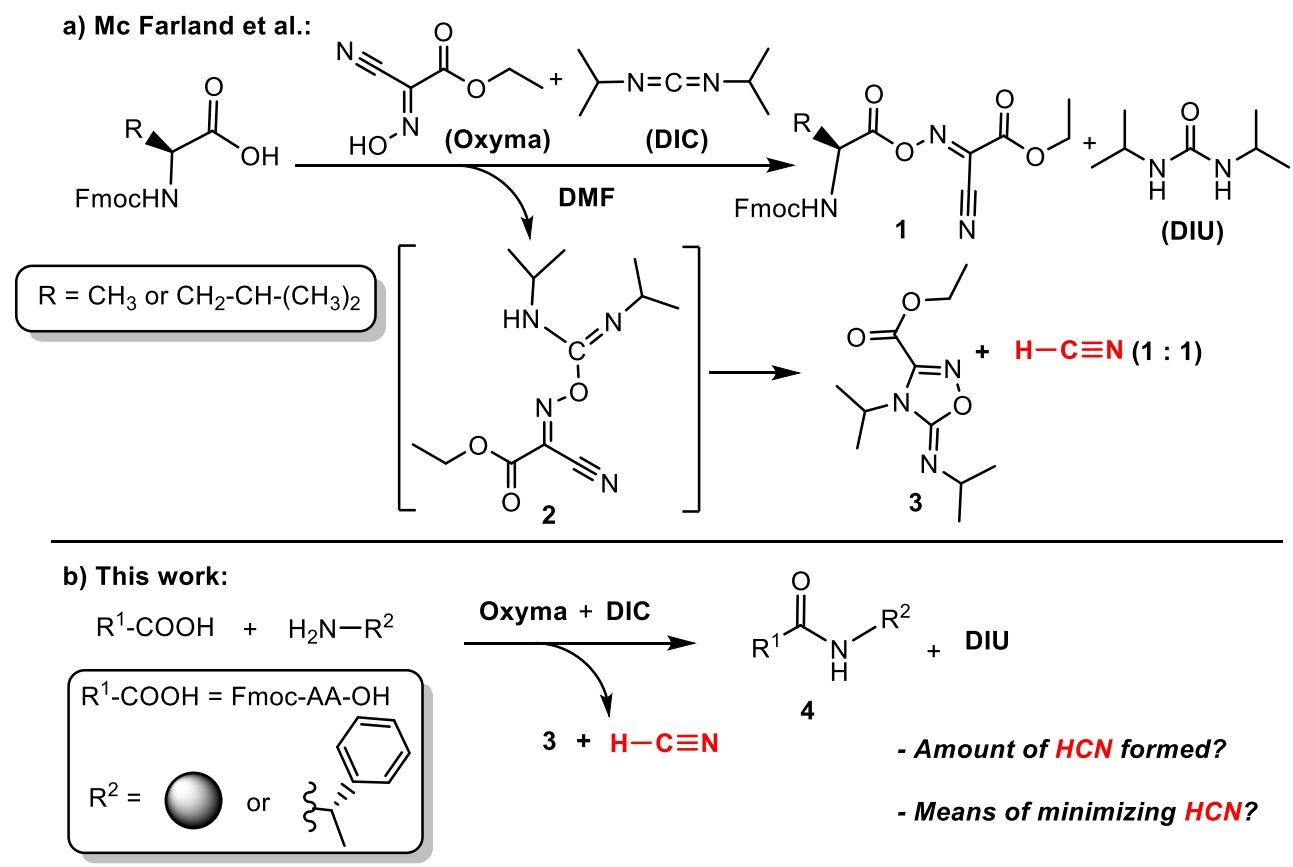

Figure 1. Schematic representation of HCN formation during DIC/Oxyma mediated reactions: (a) in reaction with Fmoc-Ala-OH or Fmoc-Leu-OH ${ }^{16}$ (b) in amide bond forming reactions and assessment of HCN minimization.

First, we set out to assess the extent of HCN formation in a model DIC/Oxyma mediated amide bond forming reaction. As oxadiazole 3 is formed in an equimolar amount as HCN in reactions involving DIC and Oxyma (Fig. 1a), ${ }^{16}$ we used the easily UVdetectable 3 as the marker of the HCN formation. As Merrifield's solid-phase peptide synthesis (SPPS) ${ }^{22}$ constitutes the most widely used method for synthesis of peptides, ${ }^{23}$ we assessed an amide bond forming reaction on a polymer support. The model system employed the Ramage linker ${ }^{24}$ bound to an aminomethylstyrene (AMS) functionalized polystyrene/divinylbenzene (PS/DVB) resin ${ }^{25}$ as nucleophile while using a Fluorenylmethyloxycarbonyl (Fmoc) protected AA activated by DIC/Oxyma as an acyl donor (Table 1). Thus, using the standard SPPS solvent DMF as the reaction medium, we carried out two amidations using AA/Oxyma/DIC starting materials in 1:1:1 and 1:1:3 stoichiometries. In both cases these starting materials were reacted for $1 \mathrm{~h}$ at room temperature $(\mathrm{rt})$ after which the activated AA solutions were added to the resin and the resulting amide bond forming reactions were allowed to proceed for $20 \mathrm{~h}$ (Table 1). The formation of the $\mathrm{HCN}$ marker 3 was monitored throughout, and $0.3 \%$ Oxyma to 3 conversion was observed (and hence equimolar amounts of HCN) after 1 h of activation of Fmoc-Ser(t-Bu)-OH/Oxyma/DIC (1:1:1) in DMF. This corroborates the results obtained by McFarland et al. who reported a similar rate of HCN formation after reacting Fmoc-Leu-OH/Oxyma/DIC (1:1:1) in DMF for $1 \mathrm{~h} .{ }^{16}$ Further to this, during the monitoring of the subsequent amide bond forming process, we found that the formation of $\mathbf{3}$ steadily increased throughout the coupling process. Although in terms of chemical conversion of Oxyma to HCN the extent of HCN formation was not significant, appreciable amounts of $\mathrm{HCN}$ would have been formed if the reaction was carried out on mol scales customary in manufacturing on industrial scale. Upon completion of these couplings as determined by a colorimetric test (ninhydrin), ${ }^{26}$ an appreciable amount of HCN was formed not only when 3 equiv DIC vs AA and Oxyma was used ( $326 \mathrm{mg} \mathrm{HCN} \mathrm{mol}^{-1}$ AA coupling) but also when 1 equiv of DIC vs AA and Oxyma was employed ( $166 \mathrm{mg} \mathrm{HCN} \mathrm{mol}^{-1} \mathrm{AA}$ coupling). It is worth noting that upon allowing the reactions to proceed further for a total of $20 \mathrm{~h}$, the content of $\mathrm{HCN}$ increased to $\sim 328 \mathrm{mg} \mathrm{HCN} \mathrm{mol}^{-1} \mathrm{AA}$ coupling for AA/Oxyma/DIC used in 1:1:1 ratio 
and to $2148 \mathrm{mg} \mathrm{HCN} \mathrm{mol}^{-1} \mathrm{AA}$ coupling for 1:1:3 ratio. This result shows that if the reactions are stopped as soon as the desired coupling conversion is attained, the total amount of formed $\mathrm{HCN}$ is reduced.

Table 1: Assessment of HCN formation during DIC/Oxyma mediated amide bond formation on solid support ${ }^{1}$

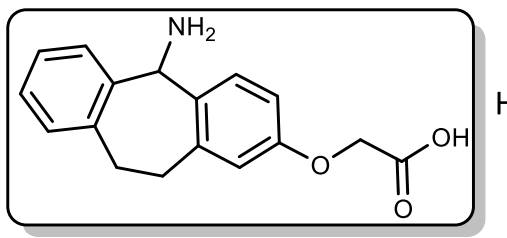

Ramage linker (H-RMG-OH)

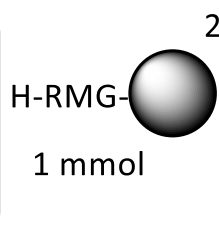

2 equiv Fmoc-Ser(t-Bu)-OH/Oxyma/

DIC $(1: 1: X$

byproducts formed per Fig. 1 :

oxadiazole $3+\mathrm{H}-\mathrm{C} \equiv \mathrm{N}(1: 1)$

\begin{tabular}{ccccccc}
\hline & \multicolumn{3}{c}{ AA activation (h) } & \multicolumn{3}{c}{ Amide bond formation (h) } \\
\cline { 2 - 6 } & 0.0 & 0.5 & 1.0 & 0.5 & 2.5 & 20 \\
\cline { 2 - 6 } & 0.00 & 0.21 & 0.28 & 0.32 & $0.48^{3}$ & 0.94 \\
\hline & 0.00 & 0.28 & 0.30 & $0.97^{3}$ & 1.66 & 6.37
\end{tabular}

${ }^{1}$ Reagents and conditions: Fmoc-Ser(t-Bu)-OH/Oxyma/DIC in DMF was reacted for $1 \mathrm{~h}$ at $\mathrm{rt}$ and the resulting mixture was added to H-RMG resin (swollen in DMF and drained). The slurry thus obtained was shaken at $\mathrm{rt}(300 \mathrm{rpm})$ for $20 \mathrm{~h} .{ }^{2}$ Aliquots of reaction mixtures were taken out throughout and analyzed by HPLC for the content of the HCN marker $\mathbf{3}$. Using these contents of $\mathbf{3}$ Oxyma to HCN conversions were determined, see section 2 in the ESI for details. ${ }^{3}$ Full conversion of the amide bond formation attained according to a qualitative (ninhydrin) color test.

As we determined that HCN formation occurs under commonly used conditions for use of DIC/Oxyma in peptide synthesis we set out to examine whether the extent of this side reaction could be minimized by suitably altering the parameters of the reaction between DIC and Oxyma. Recently, we reported that choice of solvent and/or addition of scavengers have a profound effect on the extent of side reactions initiated by conversion of Oxyma to the corresponding $\mathrm{N}$-oxyl radical. ${ }^{27}$ We reasoned that the reaction medium could alter the rate of the HCN forming side reaction as well and, thus, wanted to evaluate the solvent effects on the conversion of Oxyma to HCN. As oxadiazole 3 is directly associated with formation of HCN and the linear precursor $\mathbf{2}$ (Fig. 1a) is not, we decided to evaluate the rate of formation of both $\mathbf{3}$ and $\mathbf{2}$ in reactions between DIC/Oxyma solely and in solvents considered to be greener than the standard hazardous DMF, ${ }^{28}$ but still relevant in the context of SPPS. Two greener dipolar aprotic solvents, $\mathrm{NBP}^{29}$ and $\mathrm{PC}^{30}$ were chosen. In addition, as we recently showed that using EtOAc, a suitable solvent for greener solution-phase amidations, ${ }^{31}$ works well as a co-solvent in SPPS and even enables suppression of side reactions in peptide couplings on polymer supports, ${ }^{32}$ we also examined 1:1 mixtures of NBP/EtOAC and PC/EtOAc. With regards to scavengers, addition of a suitable sulfur containing radical scavenger such as dithiothreitol (DTT) or diisopropylthiourea (DITU) was reported to inhibit the formation of $\mathrm{N}$-Oxyl radical from Oxyma. ${ }^{27}$ Therefore, all five solvents were evaluated in the presence of DITU (10 mol\%) to discern any possible role of an N-Oxyl radical formed from Oxyma in the side reaction leading to the HCN formation. The progress of formation of $\mathbf{3}$ was assessed by HPLC over the course of 17 days, and the content of $\mathbf{2}$ and $\mathbf{3}$ was 
determined after 4 days by EIC (extracted ion chromatography) LC-MS (Fig. 2). We found that the rate of formation of 3 depends profoundly on the reaction medium (see ESI, section 3), and the 2:3 ratio could be altered by replacing DMF with any of the four greener solvents examined. Although in none of the experiments the occurrence of $\mathbf{3}$ could be minimized to an appreciable extent, we observed that the amount of 2 present increased in order of DMF NBP<NBP/EtOAc PC $<$ PC/EtOAc. It is worth noting that the open chain DIC-Oxyma adduct $\mathbf{2}$ was detected at $\mathrm{rt}$, whereas in the study by McFarland et al. it was only observed by NMR at $-30{ }^{\circ} \mathrm{C} .{ }^{16}$ Regarding the addition of $10 \mathrm{~mol} \%$ of DITU, we determined that it did not alter the 2:3 ratio appreciably irrespective of solvent (see ESI section 3 ) and this result disfavors a role of Oxyma based N-oxyl radical species in the formation of 3 and $\mathrm{HCN}$.

Having evaluated the effect of solvents on formation of adduct $\mathbf{2}$ vs oxadiazole $\mathbf{3}$ and HCN in DIC/Oxyma mixtures, a screening of actual amide bond formations on a solid support in the different solvents was initiated. Thus, we examined DMF vs the greener NBP and NBP/EtOAc (1:1), which both constitute suitable SPPS solvents. ${ }^{33,34}$ Moreover, to examine the role of NBP/EtOAc ratio on the formation of HCN, NBP/EtOAc (1:4) mixture was examined as well. With regards to PC and PC/EtOAC $(1: 1)$, these solvents were not further assessed since inferior amide bond formation kinetics were determined outside this work (unpublished data).

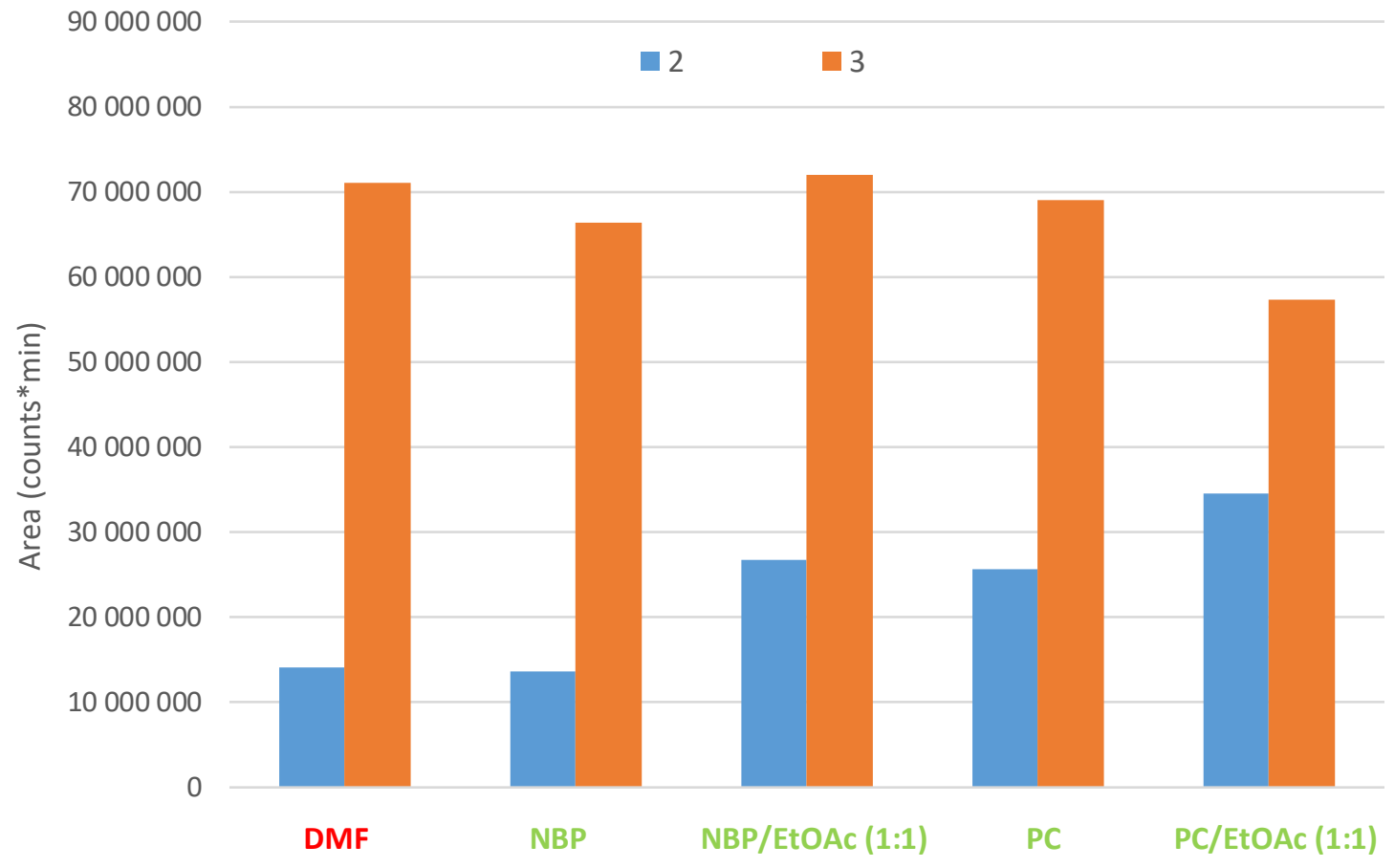

Figure 2. Schematic representation of EIC-MS contents of linear DIC/Oxyma adduct $\mathbf{2}$ and oxadiazole $\mathbf{3}$ in Oxyma/DIC (1.0:1.2) mixtures in five different solvents after 4 days at rt. For full details of HPLC analyses at different timepoints with and without 10 mol\% DITU and LC-HRMS analyses confirming the presence of $\mathbf{2}$ and $\mathbf{3}$ in these reactions see ESI, section 3.

For the coupling screening, 2 equiv. of AA/Oxyma/DIC in 1:1:1 and 1:1:3 ratios were examined. To avoid the formation of HCN during AA/DIC/Oxyma activation, the amide bond forming reactions were carried out as "one-pot", i.e. to a slurry of 
dissolved AA, Oxyma and resin, DIC was added (Table 2). The coupling conversion was assessed by a colorimetric (ninhydrin) test $^{26}$ and the formation of $\mathbf{3}$ at the completion of the experiment was determined by LC-HRMS. According to the color test carried out after $0.5 \mathrm{~h}$, all couplings using 1:1:3 AA/Oxyma/DIC were complete, while using the 1:1:1 AA/Oxyma/DIC ratio all reactions except for the NBP/EtOAc (1:4) run were incomplete. Interestingly, while the runs using 1:1:3 AA/Oxyma/DIC ratio all exhibited significant rate of 3 (and $\mathrm{HCN}$ ) formation which increased in order of NBP<DMF<NBP/EtOAC $(1: 1)<N B P / E t O A C(1: 4)$ the experiments run with 1:1:1 AA/Oxyma/DIC ratio showed a much slower rate of HCN formation, decreasing in order of DMF $>N B P>N B P / E t O A C(1: 1)>N B P / E t O A C(1: 4)$. The exact nature of all the phenomena governing the rate of HCN formation depending on solvent and reagent stoichiometry is currently not known and will be the subject of future studies.

Nevertheless, we were intrigued by the low rate of $\mathrm{HCN}$ formation and the high rate of amide bond formation observed with the AA/Oxyma/DIC (1:1:1) in NBP/EtOAC (1:4) and we decided to further evaluate reaction kinetics using this protocol. As the AA/Oxyma/DIC (1:1:1) coupling in NBP/EtOAC (1:4) using 2 equiv was quite fast, we decreased the amount of $A A$ to 1.3 equiv to slow down the coupling rate and facilitate the assessment of the kinetics. Furthermore, to also evaluate the solvent effect on the rate of $\mathrm{HCN}$ formation in a solution phase amidation, we carried out a DIC/Oxyma mediated coupling of Fmoc-Ser( $t$-Bu)-OH using (S)-(-)-1-phenylethylamine (1.0 equiv) as a nucleophile. The results of these experiments are summarized in Fig. 3, revealing that both in solution and on solid support the amidation kinetics increased in order of $D M F \sim N B P<N B P / E t O A C(1: 1)<N B P / E t O A C(1: 4)$ while the rate of HCN formation decreased in a DMF $>N B P>N B P / E t O A C(1: 1)>N B P / E t O A C(1: 4)$ order. In summary, using the inexpensive NBP/EtOAc $(1: 4)^{35}$ as solvent gave the highest reaction rate and the lowest rate of $\mathrm{HCN}$ formation both in solution and on a solid support.

Table 2: Solvent effects on HCN formation during DIC/Oxyma mediated amide bond formation on solid support ${ }^{1}$

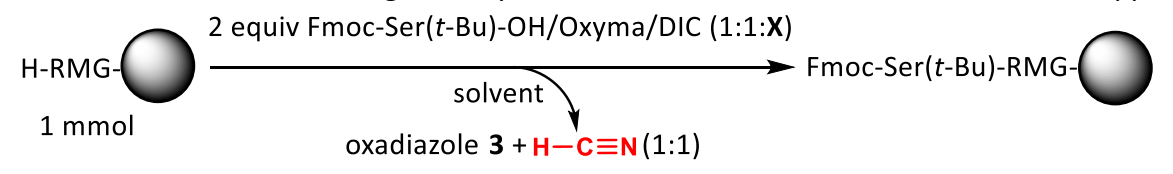

\begin{tabular}{|c|c|c|c|c|c|c|c|c|}
\hline Solvent & \multicolumn{2}{|c|}{ DMF } & \multicolumn{2}{|c|}{ NBP } & \multicolumn{2}{|c|}{ NBP/EtOAc (1:1) } & \multicolumn{2}{|c|}{ NBP/EtOAc (1:4) } \\
\hline$x$ & 1 & 3 & 1 & 3 & 1 & 3 & 1 & 3 \\
\hline \multicolumn{9}{|l|}{$\begin{array}{l}\text { Kaiser test } \\
\text { after } 0.5 \mathrm{~h}^{2}\end{array}$} \\
\hline \multicolumn{9}{|l|}{ Oxyma to } \\
\hline $\begin{array}{l}\text { HCN conv. } \\
\text { after } 20 \mathrm{~h} \\
(\%)^{3}\end{array}$ & 0.74 & 8.84 & 0.45 & 6.38 & 0.20 & 11.47 & 0.09 & 22.54 \\
\hline
\end{tabular}

${ }^{1}$ Reagents and conditions: Fmoc-Ser(t-Bu)-OH/Oxyma in solvent was added to H-RMG resin (swollen in solvent and drained). DIC was added and the slurry thus obtained was shaken at $\mathrm{rt}(300 \mathrm{rpm})$ for $20 \mathrm{~h}$. ${ }^{2}$ After $2.5 \mathrm{~h}$ the color test was negative for all runs. ${ }^{3}$ Oxyma to HCN conversions were determined as in Table 1, see section 4 in the ESI for details. 

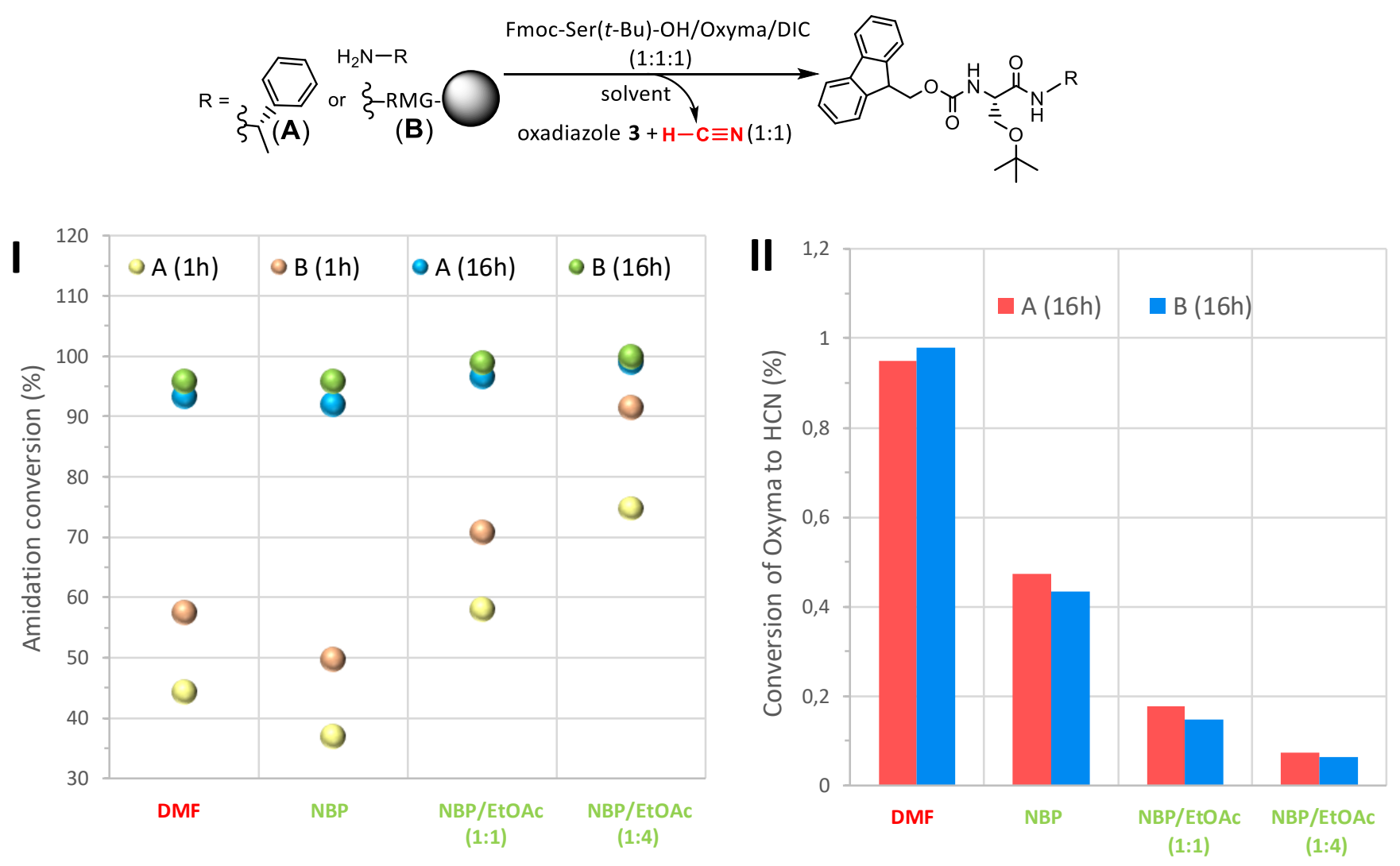

Figure 3. Solvent effects on I) amidation conversion II) HCN formation during DIC/Oxyma mediated amide bond formation in solution $(\mathrm{R}=\mathrm{A})$ and on solid support $(\mathrm{R}=\mathrm{B})$.

The encouraging results of minimizing $\mathrm{HCN}$ as well as improving reaction rates in NBP/EtOAC (1:4) represent an interesting perspective over the conventional DMF protocols. However, the occurrence of HCN was not fully eradicated. In a further pursuit of methods that would enable additional reduction of $\mathrm{HCN}$, we evaluated the concept of in situ scavenging of the HCN formed. Mc Farland et al. showed that in DMF- $\mathrm{d}_{7}$ mixtures of DIC/Oxyma, the amount of HCN in solution was appreciably lower than the amount of oxadiazole 3, conceivably due to the loss of HCN to the vapor phase above the reaction solution. ${ }^{16}$ This observation prompted us first to examine the effect of the concentration on the ratio of HCN vs $\mathbf{3}$ in solution prior to any test for HCN scavenging effects. We examined DMF- $\mathrm{d}_{7}$ solutions of DIC/Oxyma at 0.3M, 0.4M and 0.5M by NMR spectroscopy (Fig. 4) and determined the amount of $\mathrm{HCN}$ versus 3. Interestingly, a marked concentration dependence was observed. At 0.4M and 0.5M the relative amount of $\mathrm{HCN}$ ( $\mathrm{HCN}$ vs 3 ) in solution over time decreased quite significantly whereas at $0.3 \mathrm{M}$ the relative amount of HCN in solution remained much higher (Fig. 4A). The HCN lacking in solution due to loss of HCN to the gas phase would be more pronounced at higher concentrations in line with the generated results. To further test this hypothesis, future studies will be aimed at determining the content of HCN both in solution and in gas phase. ${ }^{36}$ 
A

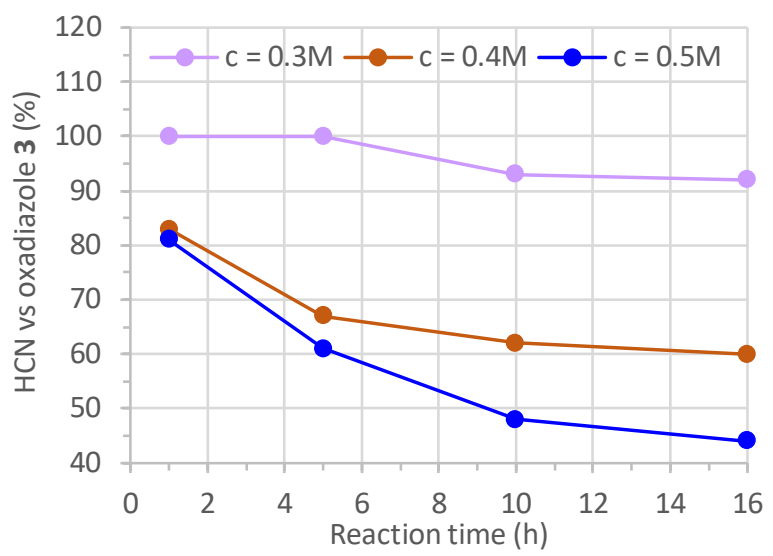

B

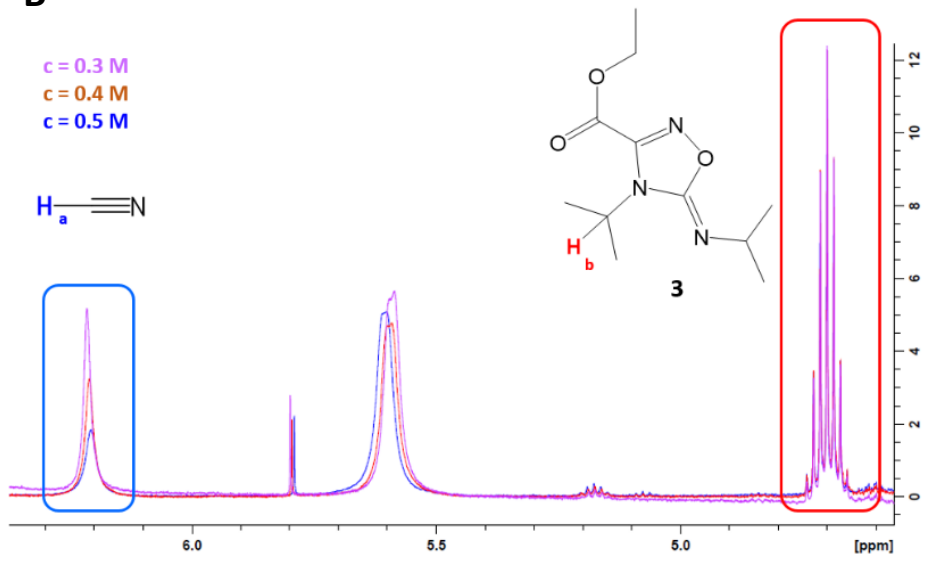

Figure 4. ${ }^{1} \mathrm{H}$ NMR assessment of the presence of $\mathrm{HCN}$ in $0.3 \mathrm{M}, 0.4 \mathrm{M}$ and $0.5 \mathrm{M}$ DMF- $\mathrm{d}_{7}$ solutions of DIC/Oxyma (1:1) at rt. A) HCN vs oxadiazole 3 ratio at different DIC/Oxyma concentrations over time. B) An expansion of an overlay of ${ }^{1} \mathrm{H}$ NMR spectra of $0.3 \mathrm{M}$, $0.4 \mathrm{M}$ and $0.5 \mathrm{M}$ DIC/Oxyma mixtures after $10 \mathrm{~h}$. An integration of $\mathrm{HCN} \mathrm{H}_{\mathrm{a}}$ and oxadiazole $3 \mathrm{H}_{\mathrm{b}}$ peaks was used to determine $\mathrm{HCN}$ vs 3 ratios, for experimental details see ESI section 7.

With the result of the DIC/Oxyma concentration study (Fig. 4) in hand we moved on to the HCN scavenger proof of concept. The concentration of the amidation reactions was chosen to be at $0.1 \mathrm{M}$ to keep the $\mathrm{HCN}$ to be scavenged in solution, and the reaction was to be monitored by NMR. As an HCN scavenger DMTS ${ }^{37}$ was employed using a reaction where DMTS transforms the HCN into the less hazardous methyl thiocyanate. Prior to engaging DMTS, it was first established that i) none of the starting materials used in DIC/Oxyma mediated amide bond formations react with the DMTS, and ii) activation of an AA by DIC/Oxyma is not impaired by the presence of DMTS (see ESI, section 8). Upon confirmation of these prerequisites, DIC/Oxyma mediated coupling of FmocGly-OH with (S)-(-)-1-phenylethylamine in DMF- $\mathrm{d}_{7}$ at $0.1 \mathrm{M}$ concentration was carried out using 0, 5 and 10 equiv. of DMTS, respectively. NMR analyses showed that increasing the amount of DMTS resulted in lowering of the HCN concentration (Fig. 5), confirming that DMTS scavenges the HCN formed during the coupling reaction. Importantly, using NMR it was determined that HCN scavenging occurred without impairing the rate of the coupling reaction (Fig. 5). Additionally, we evaluated the impact of DMTS on the rate of amide bond formations in the different solvents. Thus, DIC/Oxyma mediated couplings of Fmoc-Ser $(t-\mathrm{Bu})-\mathrm{OH}$ and (S)-(-)-1-phenylethylamine in DMF and NBP/EtOAc (1:4) were carried out, with and without 10 equiv of DMTS. In keeping with the assessment of solvents effects on coupling kinetics shown in Fig. 3 the amidations in NBP/EtOAc (1:4) occurred at faster rates than the corresponding reactions in DMF and according to HPLC analyses, the couplings with and without DMTS proceeded comparably (see ESI, section 10).

In future studies we will continue to determine the amount of $\mathrm{HCN}$ as well as rate of amide bond formation in a broader range of solvents and in the presence of various potential HCN scavengers with the aim of establishing greener, safer coupling conditions. 


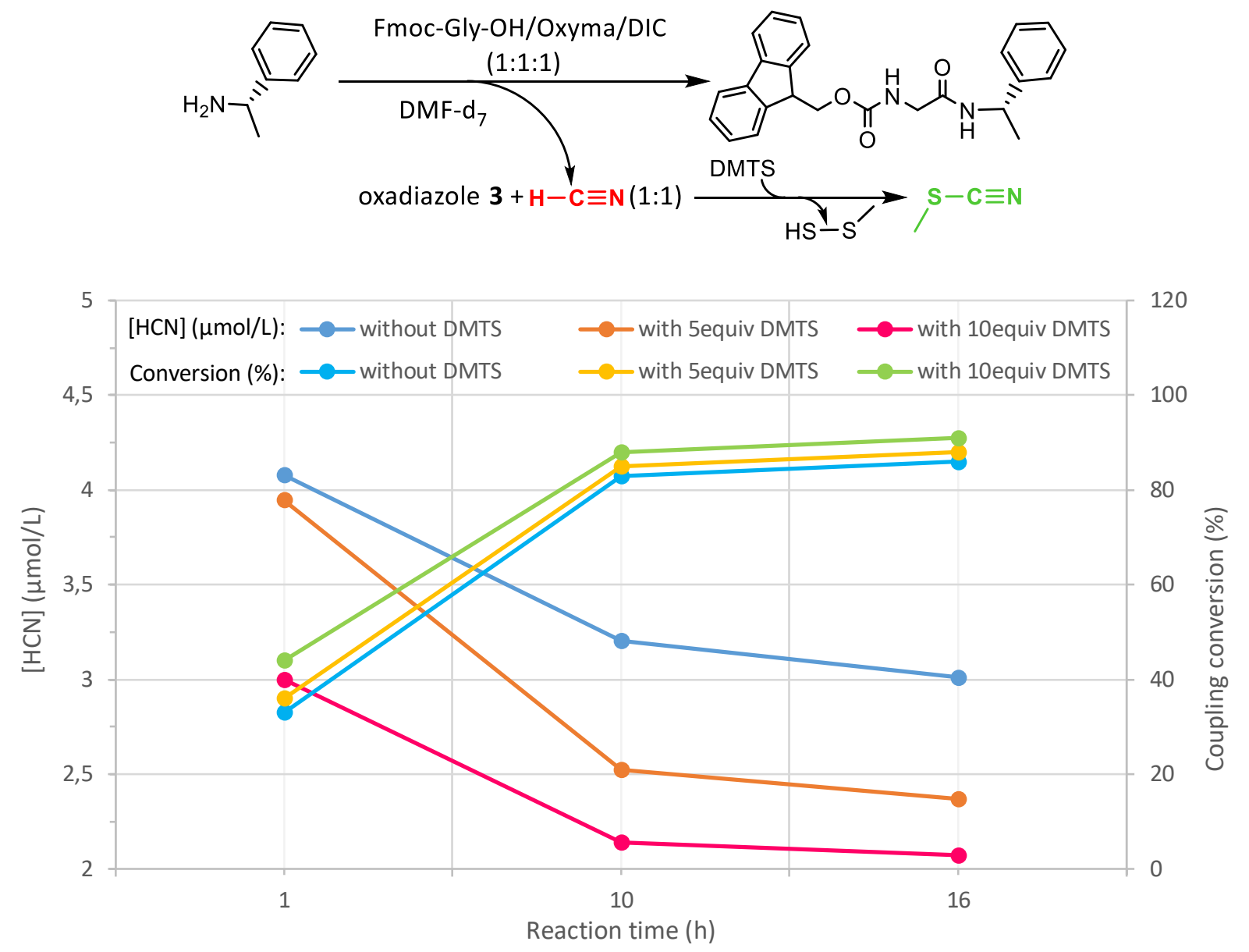

Figure 5. Effect of DMTS on conversion and presence of $\mathrm{HCN}$ during DIC/Oxyma mediated coupling of Fmoc-Gly-OH (1.0 equiv) and (S)-(-)-1-phenylethylamine in DMF- $d_{7}$ at rt.

\section{Conclusions}

In this initial work on understanding the HCN formation and identifying paths forward for control and prevention, we have demonstrated that DIC/Oxyma mediated amide bond forming reactions are accompanied by formation of HCN and that the $\mathrm{HCN}$ continues to build up in the reaction mixture. The rate of formation of $\mathrm{HCN}$ was found to be dependent on the solvent composition and the reagents ratios, and among the investigated greener alternatives, NPB/EtOAc (1:4) was found to minimize the formation while accelerating the actual coupling kinetics when employed with a 1:1:1 ratio of AA/Oxyma/DIC. The observed gap between the amounts of HCN and the amounts of 3, attributed to release of HCN into the vapor phase, was investigated and a concentration of $0.3 \mathrm{M}$ (DIC and Oxyma) was found to contain the HCN in the solution significantly more effective than higher concentrations. Moreover, the concept of HCN scavenging during the DIC/Oxyma mediated coupling reactions was successfully tested with DMTS. We propose that combining the two HCN minimization concepts involving solvent engineering and in situ $\mathrm{HCN}$ scavenging may furnish protocols for efficient DIC/Oxyma mediated, HCN free synthesis of amides and peptides in green solvents. Further work on the development of such protocols as well as their implementation in industrial manufacturing of peptides is underway and will be reported in due course.

\section{Experimental Section}

All reagents, reactants and solvents were from standard suppliers of raw materials for peptide synthesis and were used as such. 
Assessment of HCN formation and amide bond formation kinetics during DIC/Oxyma mediated coupling of 1.3 equiv FmocSer(t-Bu)-OH with H-RMG resin in different solvents.

$4 \times 1 \mathrm{mmol}$ of dry H-RMG AMS resin was prepared as described in section 2 of the ESI. The dried H-RMG AMS resins were swollen in solvents specified for each reaction and drained. The amounts of residual solvent in the swollen and drained resins were determined to be $\sim 5 \mathrm{~mL}$. Next, in four reactions vessels, 4 x $498.5 \mathrm{mg}$ (1.3 mmol, 1.0 equiv) of Fmoc-Ser(t-Bu)-OH and $184.7 \mathrm{mg}$ (1.3 mmol, 1.0 equiv) of Oxyma were dissolved in $20 \mathrm{~mL}$ of solvents as specified for each reaction. The resulting Fmoc-Ser(t-Bu)$\mathrm{OH} / \mathrm{Oxyma}$ mixtures were added to the washed and drained batches of H-RMG AMS resins prepared above. To the resulting slurries of Fmoc Ser(t-Bu)-OH/Oxyma with H-RMG AMS resins $203.9 \mu \mathrm{L}$ DIC (1.3 mmol, 1.0 equiv) was added in the following manner: experiment R1, DMF; experiment R2, NBP; experiment R3, NBP/EtOAc (1:1); experiment R4, NBP/EtOAc (1:4) and the resulting reaction mixtures were shaken at $\mathrm{rt}(300 \mathrm{rpm})$ for $16 \mathrm{~h}$. Conversions of the amidation reactions were followed by determining the Fmoc content of the Fmoc Ser(t Bu)-RMG AMS resins and EIC MS analyses were used for the determination of $\mathbf{3}$ based on which Oxyma to HCN conversions were determined (see section 5 of the ESI for details).

Assessment of HCN formation and amide bond formation kinetics during DIC/Oxyma mediated coupling of 1.0 equiv FmocSer(t-Bu)-OH with (S)-(-)-1-phenylethylamine in different solvents.

Fmoc-Ser(t-Bu)-OH (191.8 mg, $0.5 \mathrm{mmol}, 1.0$ equiv) and Oxyma (71.0 mg, $0.5 \mathrm{mmol}, 1.0$ equiv) were weighed into four glass reaction vessels equipped with a magnetic stirrer. $10.0 \mathrm{~mL}$ of a solvent (DMF, NBP, NBP/EtOAc (1:1) and NBP/EtOAc (1:4)) was added to these vessels and all four reactions thus obtained were stirred at rt until all solid material was dissolved. To the resulting reaction mixtures (S)-(-)-1-phenylethylamine (62.9 $\mu \mathrm{L}, 0.5 \mathrm{mmol}, 1.0$ equiv) and DIC (78.3 $\mu \mathrm{L}, 0.5 \mathrm{mmol}, 1.0$ equiv) were added in the following manner: experiment R1, DMF ; experiment R2, NBP; experiment R3, NBP/EtOAc (1:1); experiment R4, NBP/EtOAC (1:4) and the resulting amidation reactions were allowed to stir at $\mathrm{rt}$ for $16 \mathrm{~h}$. Conversions of the amidation reactions were followed by HPLC and EIC MS analyses were used for the determination of $\mathbf{3}$ based on which Oxyma to HCN conversions were determined (see section 6 of the ESI for details).

NMR analysis of HCN formation at different DIC/Oxyma concentrations

A solution was prepared by dissolving Oxyma $(7.2 \mathrm{mg}, 0.051 \mathrm{mmol}, 1.0$ equiv) and caffeine as internal standard ( $2.1 \mathrm{mg}, 0.011$ mmol, 0.22 equiv) in varying amount of DMF-d7 (see ESI section 7) to obtain $0.3,0.4$ and $0.5 \mathrm{M}$ concentration of Oxyma. The solution was mixed by using an ultrasound bath and $\mathrm{DIC}(8.0 \mu \mathrm{L}, 0.051 \mathrm{mmol}, 1.0$ equiv) was added and the resulting mixture was transferred into a $3 \mathrm{~mm} \mathrm{NMR}$ tube and transferred to the spectrometer for monitoring at $20^{\circ} \mathrm{C}$. 1D ${ }^{1} \mathrm{H}$ NMR acquisition was done after $1 \mathrm{~h}, 5 \mathrm{~h}, 10 \mathrm{~h}$ and $16 \mathrm{~h}$ at which times HCN content in the solutions were determined (see ESI section 7).

NMR analysis of HCN formation during amide bond formations mediated by DIC/Oxyma with and without DMTS

Oxyma (10.1 mg, 0.071 mmol, 1.04 equiv), Fmoc-Gly-OH (20.3 mg, $0.068 \mathrm{mmol}, 1.0$ equiv) and caffeine (8.0 mg, $0.042 \mathrm{mmol}, 0.62$ equiv) were dissolved in $680 \mu \mathrm{L}$ of DMF-d7 and (S)-(-)-1-phenylethylamine (8.77 $\mu \mathrm{L}, 0.068 \mathrm{mmol}, 1.0$ equiv) and DIC (10.65 $\mu \mathrm{L}$, $0.068 \mathrm{mmol}, 1.0$ equiv) were added. $170 \mu \mathrm{L}$ aliquots ( $25 \% \mathrm{v} / \mathrm{v}$ of the solution i.e. $\sim 0.017 \mathrm{mmol}$ based on $\mathrm{Fmoc}-\mathrm{Gly} \mathrm{OH}$ ) of this solution were taken out and $0,5,10$ equiv of DMTS were added to these solutions. The resulting reaction mixtures were transferred into $3 \mathrm{~mm}$ NMR tubes and 1D ${ }^{1} \mathrm{H}$ NMR acquisitions were done after $1 \mathrm{~h}, 5 \mathrm{~h}, 10 \mathrm{~h}$ and $16 \mathrm{~h}$ using which amidation conversions and contents of HCN were determined (see ESI section 9).

Conflicts of interest

There are no conflicts to declare.

References 
1 Anastas, P. T.; Zimmerman, J. B., The United Nations sustainability goals: How can sustainable chemistry contribute? Curr. Opin. Green Sustainable Chem. 2018, 13, 150.

${ }^{2}$ Erythropel, H. C.; Zimerman, J. B.; de Winter, T. M.; Petitjean, L.; Melnikov, F.; Lam, C. H.; Lounsbury, A. W.; Mellor, K. E.; Janković, N. Z.; Tu, Q.; Pincus, L. N.; Falinski, M. M.; Shi, W.; Coish, P.; Plata, D. L.; Anastas, P. T., The Green ChemisTREE: 20 years after taking root with the 12 principles. Green Chem. 2018, 20, 1929.

${ }^{3}$ See e.g. a) Canavelli,P.; Islam, S.; Powner, M. W., Peptide ligation by chemoselective aminonitrile coupling in water. Nature, 2019, 571, 546; b) Henninot, A.; Collins, J. C.; Nuss, J. M. The Current State of Peptide Drug Discovery: Back to the Future? J. Med. Chem. 2018, 61, 1382.

${ }^{4}$ a) Bryan, M. C.; Dunn, P. J.; Entwistle, D.; Gallou, F; Koenig, S. G.; Hayler, J. D.; Hickey, M. R.; Hughes, S,; Kopach, M. E.; Moine, G.; Richardson, P.; Roschangar, F.; Steven, A.; Weiberth, F. J., Key Green Chemistry research areas from a pharmaceutical manufacturers' perspective revisited. Green Chem. 2018, 20, 5082; b) Llobet, A. I.; Kenworthy, M. N.; Mukherjee, S.; Kopach, M. E.; Wegner, K.; Gallou, F.; Smith, A. G.; Roschangar, F., Sustainability Challenges in Peptide Synthesis and Purification: From R\&D to Production. J. Org. Chem. 2019, 84, 4615; c) Amide Bond Formation Strategies: Latest Advances on a Dateless Transformation. Eur. J. Org. Chem. 2020, 21, DOI: /10.1002/ejoc.202000080.

${ }^{5}$ a) Sabatini, M. T.; Boulton, L. T.; Sneddon, H. F.; Sheppard, T. D., A green chemistry perspective on catalytic amide bond formation. Nature Cat. 2019, 2, 10; b) Wang, X., Challenges and outlook for catalytic direct amidation reactions Nature Cat. 2019, 2, 98.

${ }^{6}$ de Figueiredo, R. M. ; Suppo, J.-S.; Campagne, Nonclassical Routes for Amide Bond Formation. J.-M. Chem. Rev. 2016, 116, 12029.

${ }^{7}$ El-Faham, A.; Albericio, F., Peptide Coupling Reagents, More than a Letter Soup. F., Chem. Rev. $2011,111,6557$.

${ }^{8}$ See e.g. a) Sperry, J. B.; Minteer, J. B.; Tao, J.; Johnson, R.; Duzguner, R.; Hawksworth, M.; Oke, S.; Richardson, P. F.; Barnhart, R.; Bill, D. R.; Giusto, R. A.; Weaver III, J. D., Thermal Stability Assessment of Peptide Coupling Reagents Commonly Used in Pharmaceutical Manufacturing. Org. Process Res. Dev. 2018, 22, 1262; b) Treitler, D. S.; Marriott, A. S.; Chadwick, J.; Quirk, E., Mutagenic Impurities in 1-Hydroxybenzotriazole (HOBt). Org. Process Res. Dev. 2019, 23, 2562; c) McKnelly, K. J.; Sokol, W.; Nowick, J. S., Anaphylaxis Induced by Peptide Coupling Agents: Lessons Learned from Repeated Exposure to HATU, HBTU, and HCTU. J. Org. Chem. 2020, 85, 1764.

${ }_{9}^{9}$ Benoiton, N. L.; Chen, F. M., Not the alkoxycarbonylamino-acid O-acylisourea. J. Chem. Soc., Chem. Commun., 1981, 543.

${ }^{10}$ El-Faham, A.; Albericio, F., Synthesis and Application of N-Hydroxylamine Derivatives as Potential Replacements for HOBt. Eur. J. Org. Chem. 2009, 10, 1499.

${ }^{11}$ Albericio, F.; El-Faham, A., Choosing the Right Coupling Reagent for Peptides: A Twenty-Five-Year Journey. Org. Process Res. Dev. 2018, 22, 760.

${ }^{12}$ Fattahi, N.; Ayubi, M.; Ramazani, A., Amidation and esterification of carboxylic acids with amines and phenols by $\mathrm{N}, \mathrm{N}^{\prime}$-diisopropylcarbodiimide: A new approach for amide and ester bond formation in water. Tetrahedron Lett. 2018, $74,4351$.

13 Jad, J. E.; Khattab, S. N.; de la Torre, B. G.; Govender, T.; Kruger, H. G.; El-Faham, A.; Albericio, F., Oxyma-B, an excellent racemization suppressor for peptide synthesis. Org. Biomol. Chem. 2014, 12, 8379.

${ }^{14}$ Subirós-Funosas, R.; Prohens, R.; Barbas, R.; El-Faham, A.; Albericio, F., Oxyma: An Efficient Additive for Peptide Synthesis to Replace the Benzotriazole-Based HOBt and HOAt with a Lower Risk of Explosion. Chem. Eur. J. 2009, 15, 9394.

${ }^{15}$ Wehrstedt, K. D.; Wandrey, P. A.; Heitkamp, D., Explosive properties of 1-hydroxybenzotriazoles. J. Hazard. Mater. 2005, 126, 1.

${ }^{16}$ McFarland, A. D.; Buser, J. Y.; Embry, M. C.; Held, C. B.; Kolis, S. P., Generation of Hydrogen Cyanide from the Reaction of Oxyma (Ethyl cyano(hydroxyimino) acetate) and DIC (Diisopropylcarbodiimide). Org. Process Res. Dev. 2019, 23, 2099.

17 Patel, B. H.; Percivalle, C.; Ritson, D. J.; Duffy, C. D.; Sutherland, J. D., Common origins of RNA, protein and lipid precursors in a cyanosulfidic protometabolism. Nature Chem. 2015, 7, 301.

${ }^{18}$ Udhayakumari, D., Chromogenic and fluorogenic chemosensors for lethal cyanide ion. A comprehensive review of the year 2016. Sens. Actuators, B, 2018, 259, 1022. 
19 CDC, hydrogen cyanide, in: NIOSH Pocket Guide to Chemical Hazards, retrieved from https://www.cdc.gov/niosh/npg/npgd0333.html, on May 14th 2020.

${ }^{20}$ Albericio, F.; Al Musaimi, O.; de la Torre, B. G., Greening Fmoc/tBu solid-phase peptide synthesis. Green Chem. 2020, 22, 996.

${ }^{21}$ Rasmussen, J. H.; Pawlas, J.; Ludemann-Hombourger, O.; Rekai, E. D., Aspects of greening peptide chemistry within the pharmaceutical manufacturing industry. Chem. Today, 2019, 37, 42.

${ }^{22}$ Merrifield, R. B., Solid Phase Peptide Synthesis. I. The Synthesis of a Tetrapeptide. J. Am. Chem. Soc., 1963, 85, 2149.

${ }^{23}$ Petrou, C.; Sarigiannis, Y., Peptide synthesis: Methods, trends, and challenges. Peptide Applications in Biomedicine, Biotechnology and Bioengineering. S. Koutsopoulos, ed. Woodhead Publishing, Duxford, United Kingdom, $2018,1$.

${ }^{24}$ Ramage, R.; Irving, S. L.; McInnes, C. Design of a versatile linker for solid phase peptide synthesis: Synthesis of Cterminal primary/seconary amides and hydrazides. Tetrahedron Lett. 1993, 34, 6599.

${ }^{25}$ Yan, B., The properties of resin supports and their effects on solid-phase organic synthesis. Comb. Chem. High Throughput Screen. 1998, 1, 215.

${ }^{26}$ Kaiser, E.; Colescott, R. L.; Bossinger, C. D.; Cook, P. I., Color test for detection of free terminal amino groups in the solid-phase synthesis of peptides. Anal. Biochem. 1970, 34, 595.

27 Pawlas, J.; Rasmussen, J. H., ReGreen SPPS: enabling circular chemistry in environmentally sensible solid-phase peptide synthesis Green Chem. 2019, 21, 5990.

${ }^{28}$ Byrne, F. P.; Jin, S.; Paggiola, G.; Petchey, T. H. M.; Clark, J. H.; Farmer, T. J.; Hunt, A. J.; McElroy, C. R.; Sherwood, J.; Tools and techniques for solvent selection: green solvent selection guides. Sustain. Chem. Process. $2016,4,1$.

${ }^{29}$ Sherwood, J.; Parker, H. L.; Moonen, K.; Farmer, T. J.; Hunt, A. J., N-Butylpyrrolidinone as a dipolar aprotic solvent for organic synthesis. Green Chem. 2016, 18, 3990.

30 a) Luciani, L.; Goff, E.; Lanari, D.; Santoro. S.; Vaccaro, L. Waste-minimised copper-catalysed azide-alkyne cycloaddition in Polarclean as a reusable and safe reaction medium. Green Chem. 2018, 20, 183; b) Cseri, L.; Szekely, G., Towards cleaner PolarClean: efficient synthesis and extended applications of the polar aprotic solvent methyl 5(dimethylamino)-2-methyl-5-oxopentanoate. Green Chem. 2019, 21, 4178.

${ }^{31}$ MacMillan, D. S.; Murray, J.; Sneddon, H. F.; Jamieson, C., Watson, A. J. B., Evaluation of alternative solvents in common amide coupling reactions: replacement of dichloromethane and N,N-dimethylformamide. Green. Chem. 2013, 15, 596.

${ }^{32}$ Pawlas, J., Investigating racemization in HIS couplings in SPPS. Proceedings of the $34^{\text {th }}$ European Peptide Symposium and the $8^{\text {th }}$ International Peptide Symposium, A. Beck-Sickinger, K. Mörl, K. Bellmann-Sickert, S. Els-Heindl eds., Leipzig, Germany, 2016, p. 60.

${ }^{33}$ For use of NBP as SPPS solvent see: a) Lopez, J.; Pletscher, S.; Aemissegger, A.; Bucher, C.; Gallou, F., Org. Process Res. Dev. N-Butylpyrrolidinone as Alternative Solvent for Solid-Phase Peptide Synthesis. 2018, 22, 494; b) de la Torre, B. G.; Kumar, A.; Alhassan, M.; Bucher, C.; Albericio, F.; Lopez, J., Successful development of a method for the incorporation of Fmoc-Arg(Pbf)-OH in solid-phase peptide synthesis using N-butylpyrrolidinone (NBP) as solvent. Green. Chem. 2020, 61, DOI: 10.1039/C9GC03784E.

${ }^{34}$ For use of NBP/EtOAc as SPPS solvent see: a) Pawlas, J.; Nilsson, M.; Antonic, B.; Svensson, T.; Finnman, J.; Rasmussen, J. H., TamiSolve ${ }^{T M} \mathrm{NxG}$ as a suitable solvent for cost-efficient green SPPS. Poster PM1 at TIDES, Boston, MA, U.S.A, May 2018; https://www.polypeptide.com/wp-content/uploads/2019/10/Suitable-Solvent-for-costefficient-green-SPPS_TIDES2018-PM1.pdf; b) Pawlas, J.; Antonic, B.; Lundqvist, M.; Svensson, T.; Finnman, J.; Rasmussen, J. H., 2D green SPPS: green solvents for on-resin removal of acid sensitive protecting groups and lactamization. Green Chem. 2019, 21, 2594.

${ }^{35}$ For a comparison of the cost of DMF, NBP and NBP/EtOAC as SPPS solvents see ref. 34b.

${ }^{36}$ For examples of methods suitable for determining content of HCN in gas phase and in solution see e.g ref. 18 and a) Gotor, R.; Costero, A. M.; Gil, S.; Parra, M.; Martínez-Máñez, R.; Sancenón, F.; Gaviña, P., Selective and sensitive chromogenic detection of cyanide and $\mathrm{HCN}$ in solution and in gas phase. Chem. Commun. 2013, 49, 5669; b) Greenawald, L. A.; Snyder, J. L.; Fryc, N. L.; Sailor, M. J.; Boss, G. R.; Finklea, H. O.; Bell, S., Development of a cobinamide-based end-of-service-life indicatorfor detection of hydrogen cyanide gas. Sens. Actuators, B, 2015, 221, 379.

${ }^{37}$ a) Rockwood, G. A.; Thompson, D. E.; Petrikovics, I., Dimethyl trisulfide: A novel cyanide countermeasure. Toxicol. Ind. Health, 2016, 32, 2009; b) Bhadra, S.; Zhang, Z.; Zhou, W., Ochieng, F.; Rockwood, G. A.; Lippner, D.; Logue, B. 